\title{
A importância do contexto no desenvolvimento do framework de Colaboração em MOOCs - MOOColab
}

\author{
Ana Carla A. Holanda ${ }^{1}$, Patrícia, C. A. R. Tedesco ${ }^{1 .}$ \\ ${ }^{1}$ Universidade Federal de Pernambuco (UFPE) \\ Caixa Postal 50.540-740 - Recife - PE - Brasil \\ \{acah@cin.ufpe.br, pcart@cin.ufpe.br\}
}

\begin{abstract}
The popularity of MOOCs has shortened time and space barriers in traditional education and has become an important source of access to quality resources. However, the increase in the number of students accessing such platforms with different levels of knowledge, goals and needs hinders their interaction in the environment, due to the different contexts where students are inserted. The construction of a People Recommendation System can be an alternative for the promotion of collaborative learning. Thus, this article addresses the importance of evaluating the student's context in order to recommend other students thus encouraging shared learning built from different perspectives.
\end{abstract}

\begin{abstract}
Resumo
A popularidade dos MOOCs tem limitado as barreiras de tempo e espaço na educação tradicional e tem se tornado um importante fonte de acesso a recursos de qualidade. Entretanto, o aumento do número de alunos na plataforma com diferentes niveis de conhecimentos, metas e necessidades dificulta a interação no ambiente, devido a diferentes contextos onde os alunos estão inseridos. A construção de um Sistema de Recomendação (SR) de pessoas pode ser uma alternativa para a promoção da aprendizagem colaborativa. Assim, esse artigo aborda a importância em avaliar o contexto do aluno visando recomendar outros alunos em um SR, incentivando assim, a aprendizagem compartilhada e construida a partir de diferentes olhares $e$ perspectivas.
\end{abstract}

\section{Introdução}

MOOCs (Massive Open Online Courses) vêm ganhando atenção, especialmente nos últimos anos, por ser capaz de servir dezenas de milhares de aprendizes de uma só vez, através de ferramentas colaborativas e fornecendo material didático de qualidade (CASTILLO et al., 2015). Várias plataformas como Edx ${ }^{1}$, Udacity ${ }^{2}$ e $U d e m y^{3}$ passam a serem utilizados por universidades conceituadas visando tornar a educação acessível a todos (BANSAL, 2013), incluindo muitos tipos de recursos, como vídeos, textos, teste, fóruns, chats, entre outros.

\footnotetext{
${ }^{1} \mathrm{http}: / / \mathrm{www} . \mathrm{edx} . \mathrm{org}$

${ }^{2}$ http://www.udacity.com

3 http://www.udemy.com
} 
VII Congresso Brasileiro de Informática na Educação (CBIE 2018)

Anais do XXIX Simpósio Brasileiro de Informática na Educação (SBIE 2018)

No entanto, uma das principais dificuldades relacionadas aos MOOCs é a diversidade de aprendizes. Na verdade, o mesmo curso pode ser seguido por um grande número de alunos em todo o mundo com diferentes idades, competências e objetivos, o que torna difícil a adequação dos cursos e a aprendizagem colaborativa devido à diversidade de conhecimentos prévios e metas individuais.

É, então, necessário que haja comunicação e colaboração contínua entre pares, professores e experts sendo necessário destacar a necessidade de análise do contexto das possibilidades de interação: desde as técnicas disponíveis para cada aluno, dos interesses e necessidades individuais de cada um; da possibilidade de disposição de colaboração de cada um; dos interesses e influências culturais dos envolvidos no processo de aprendizagem, além do conhecimento, experiências e expertises de cada ator no processo de colaboração. Desta forma, esta pesquisa tem como objetivo identificar a arquitetura de contexto do framework de colaboração para MOOCs - MOOColab (que está em processo de desenvolvimento) para gerar aprendizagem colaborativa neste ambiente.

Assim, este artigo está organizado da seguinte forma: a seção 2 aborda o método adotado na pesquisa; a seção 3 mostra o estado atual do trabalho; e, por fim, as considerações finais e trabalhos futuros são explicitados.

\section{Metodologia}

Esta pesquisa vai utilizar uma abordagem de estudo de caso exploratório e interpretativo, segundo o preconizado por Merriam (2009). Inicialmente foi realizada uma Revisão Sistemática da Literatura para encontrar as lacunas existentes no que se refere à colaboração em MOOCs. Foi, então, proposto o framework de colaboração MOOColab, tendo em vista o resultado da RSL e o planejamento da arquitetura do contexto através de informações contextuais para recomendação de pessoas que possam colaborar entre si e proporcionar um maior engajamento e motivação para a construção e compartilhamento do conhecimento. A implementação deste modelo está em construção. Este deverá ser integrado ao ambiente MOOC do IFAC.

\section{Estado atual do trabalho}

Em Holanda e Tedesco (2017) são apresentados os resultados uma Revisão Sistemática da Literatura (RSL) visando identificar potenciais lacunas de pesquisa em MOOCs. Vários trabalhos abordam a necessidade da colaboração no ambiente MOOC. Aher e Lobo (2013) usam técnicas de mineração de dados, como algoritmo de clustering e regra de associação para obter lista de recomendação de cursos. Kla (2011) cria um modelo de recomendação personalizado com base no estilo de aprendizagem, conhecimento e preferências dos usuários para realizar recomendação de material didático. Assim, percebeu-se que os trabalhos relacionados têm por objetivo a recomendação de cursos e materiais.

Uma vez que a abordagem de recomendação de pessoas é algo pouco explorado nas pesquisas voltadas a MOOC foram concentrados esforços na definição de um framework que incentiva a colaboração para cursos massivos tendo o objetivo de auxiliar os alunos a localizar pessoas com quem possam colaborar e gerar a possibilidade de construção da aprendizagem a partir dessas trocas de experiências e expertises.

Desta forma, foram mapeadas informações para criar um modelo do aluno e, assim, gerar recomendações que atendam às necessidades e metas dos alunos levando em 
VII Congresso Brasileiro de Informática na Educação (CBIE 2018)

Anais do XXIX Simpósio Brasileiro de Informática na Educação (SBIE 2018)

consideração o contexto em que o mesmo está inserido. Foi utilizado o processo CSS Designer Process proposto por Vieira e colegas (2009) para capturar, processar, utilizar e disseminar o contexto no ambiente de ensino escolhido. A Figura 1, abaixo, mostra a visão de arquitetura de aquisição e utilização do contexto durante a realização de um curso MOOC personalizado.

A identificação do contexto levará em consideração informações definidas pelos alunos através de questionários disponibilizados na plataforma, bem como dados de logs gerados pela própria plataforma, que vai possibilitar a criação de perfis de usuários (que podem ser modificados a partir do comportamento do aluno no ambiente).

No Gerenciamento do Contexto teremos regras estabelecidas a partir dos dados coletados e, através de inferências, será gerada uma lista de recomendações personalizadas de pessoas para facilitar o processo de comunicação e interação no ambiente MOOC. Na etapa da de Utilização do Contexto, a lista será disponibilizada no ambiente e, posteriormente, haverá uma avaliação realizada pelos alunos para identificarmos a satisfação da personalização da plataforma de acordo com as suas características e necessidades. Essa avaliação vai gerar um ranking de colaboração baseado nas interações realizadas no ambiente.

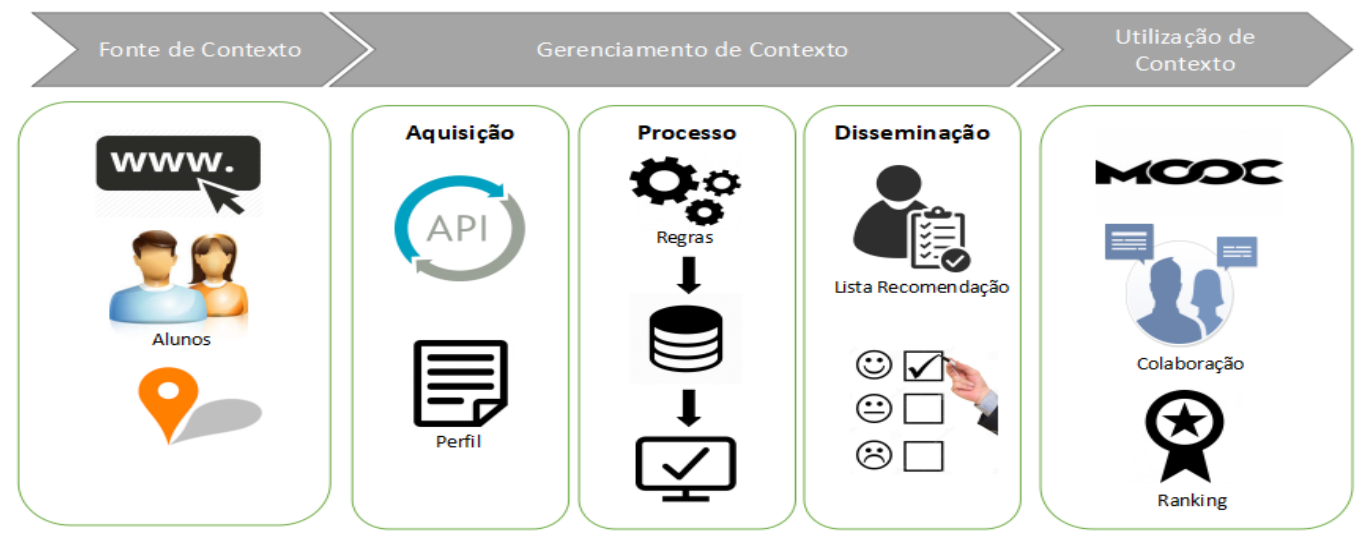

Figura 1: Visão da arquitetura do contexto no ambiente

A Figura 2 mostra como ocorre o fluxo de informações do contexto no framework. Assim que o aluno se inscreve no curso ele responde a um conjunto de perguntas iniciais para identificar o seu perfil, tomando como referência o modelo MBTI (Myers-Briggs Type Indicator) que pode ser alterada a partir da sua interação no ambiente MOOC. Ao iniciar o curso, atividades, Recursos Educacionais Abertos (REAs) e vídeos serão propostos e, a partir das ações do aluno dentro do ambiente, o sistema perceberá se este está tendo algum tipo de dificuldade no decorrer do curso, ou o próprio aluno solicitará auxílio. A partir desse momento, o sistema avaliará as informações de contexto como conhecimentos prévios, experiência, frequência de interação no ambiente, motivação, e mídias mais utilizadas, e, então, gerará uma lista de recomendação de pessoas que estejam aptas a auxiliar o aluno naquela dificuldade específica do curso. Esta lista estará ordenada de acordo com a função de aptidão descrita abaixo.

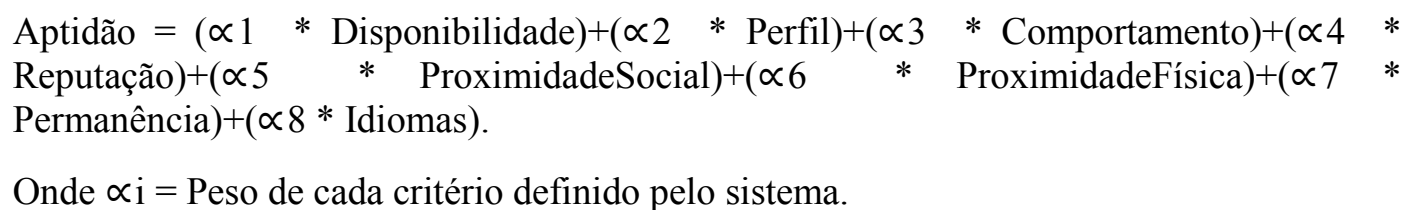


VII Congresso Brasileiro de Informática na Educação (CBIE 2018)

Anais do XXIX Simpósio Brasileiro de Informática na Educação (SBIE 2018)

O sistema irá gerar uma base de casos com informações sobre casos de indicação anteriores. É realizada, então, uma análise da similaridade entre o contexto do aluno armazenado na base de casos e o contexto do aluno que necessita de auxílio. Após a identificação da aptidão do aluno, estes são ordenados de forma crescente pela aptidão. A partir dessa análise é, então, gerado uma lista de alunos recomendados a iniciar o processo de comunicação e troca de experiências dentro do ambiente (visto na Figura 2 abaixo).

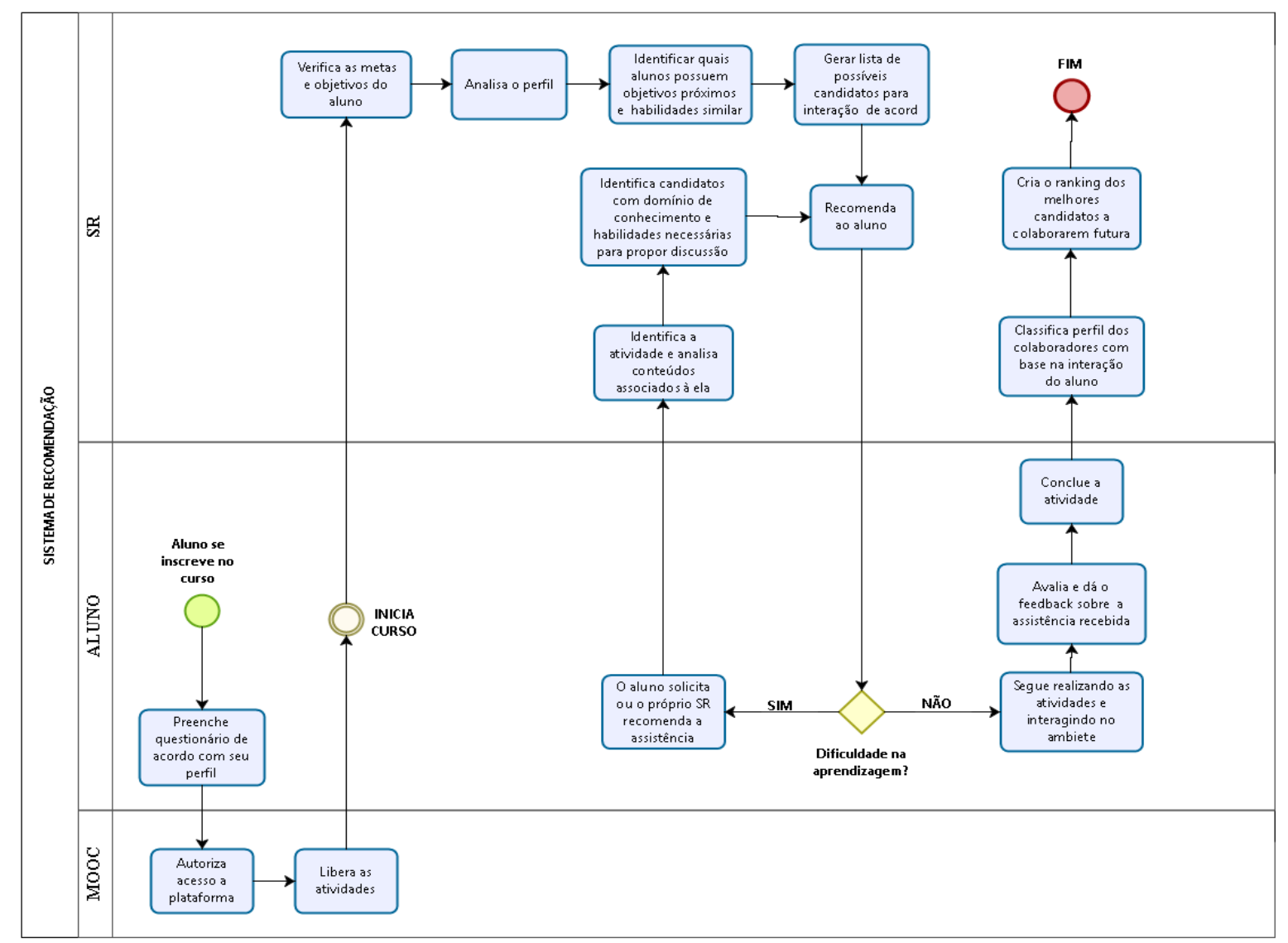

Figura 2: Fluxo de ação do framework

Para avaliar o resultado da utilização do Sistema de Recomendação será realizado um experimento com dois grupos de alunos: um grupo de controle que realizará um curso no ambiente sem a intervenção do Sistema de Recomendação desenvolvido; e um grupo experimental que terá acesso a todas as funcionalidades do Sistema de Recomendação descritas acima.

Para realizar essa avaliação será levado em consideração os estudos de Bloom (1993) que defende que a avaliação do processo ensino-aprendizagem apresenta três funções: a avaliação diagnóstica e tem como principal objetivo verificar o conhecimento prévio de cada aluno e, assim, constatar os pré-requisitos necessários de conhecimento ou habilidades imprescindíveis de que os alunos possuem para o preparo de uma nova etapa de aprendizagem. Já a avaliação formativa visa, basicamente, avaliar se o aluno domina gradativamente e hierarquicamente cada etapa da aprendizagem, antes de avançar para outra etapa subsequente de ensino-aprendizagem. 
VII Congresso Brasileiro de Informática na Educação (CBIE 2018)

Anais do XXIX Simpósio Brasileiro de Informática na Educação (SBIE 2018)

Por fim, será realizada a avaliação somativa, tem como função básica a classificação dos alunos, sendo realizada ao final do curso e, então, classificando os alunos de acordo com os níveis de aproveitamento previamente estabelecidos.

A Figura 3 sintetiza a forma de avaliação que será realizada.

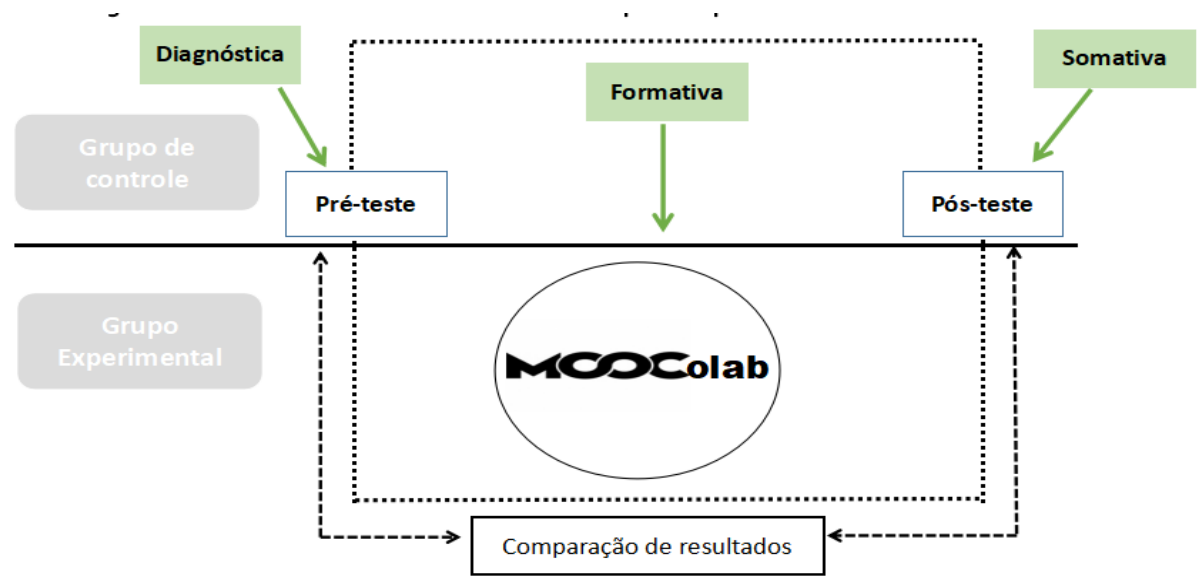

Figura 3: Avaliação da pesquisa

\section{Conclusão}

Como resultado é esperado que o framework de colaboração proporcione um ambiente personalizado, levando em consideração, o contexto que o aluno está inserido, para que as recomendações estejam alinhadas aos interesses individuais e coletivos, gerando uma aprendizagem mais colaborativa e ambientes mais adaptados às expectativas de aprendizagem dos alunos.

Atualmente o Sistema de Recomendação está em processo de desenvolvimento. A validação do trabalho também já está sendo realizada através de um experimento em um grupo que não terá a intervenção do Sistema de Recomendação para que se possa avaliar se o grupo de controle com a intervenção do Sistema personalizado apresentará melhores resultados de aprendizagem. Como trabalhos futuros, tem-se a necessidade de realização de um experimento para verificar a eficiência do framework proposto.

\section{Referências}

AHER S. B.; LOBO L. Combination of machine learning algorithms for recommendation of courses in E-Learning System based on historical data. Knowledge-Based Systems. 2013.

HOLANDA, A. C. A.; TEDESCO, P. C. A. R. MOOCs e Colaboração: definição, desafios, tendências e perspectivas. XXVIII Simpósio Brasileiro de Informática na Educação - SBIE. Congresso Brasileiro de Informática na Educação - CBIE 2018, Recife - PE, Brasil.

KLA, A. (2011). Computers \& Education E-Learning personalization based on hybrid recommendation strategy and learning style identification. 885-899. https://doi.org/10.1016/j.compedu.2010.11.001

VIEIRA, V; SOUZA, D; SALGADO, A. C.; TEDESCO, Patrícia. Modelos e processos para o desenvolvimento de Sistemas Sensíveis ao Contexto. JAI - XXVIII Jornadas de Atualização em Informática, XXIX. Congresso da Sociedade Brasileira de Computação, 2009. 